\title{
On a Subclass of Harmonic Mappings Associated with Hypergeometric Functions
}

\author{
R. Chandrashekar ${ }^{1^{*}}$, See Keong Lee ${ }^{2}$ and Pradeep Isawasan 3 \\ ${ }^{1}$ Faculty of Technology Management and Business, Universiti Tun Hussein Onn Malaysia, 86400 \\ Parit Raja, Batu Pahat, Johor, Malaysia \\ ${ }^{2}$ School of Mathematical Sciences, Universiti Sains Malaysia, 11800 USM, Penang, Malaysia \\ ${ }^{3}$ Department of Computer Science, Universiti Tunku Abdul Rahman (UTAR), 31900 Kampar, \\ Perak, Malaysia
}

\begin{abstract}
$H P(\alpha, \beta)$ is a class of functions harmonic and univalent defined in the open unit disc $U$. Sufficient conditions for a hypergeometric function and an integral operator related to hypergeometric function, to be in the class $H P(\alpha, \beta)$ are derived. Harmonic functions with negative coefficients are also considered in this investigation.
\end{abstract}

Keywords: Harmonic functions; hypergeometric functions; convolution; integral operator

\section{INTRODUCTION}

The basic theory of harmonic mappings was initiated in the seminal works of Clunie and Sheil-Small (1984) and Sheil-Small (1990). Since then harmonic univalent functions have been intensively investigated from the point of view of geometric function theory. See for example (Ahuja, 2005; Duren, 2004; Liu \& Ponnusamy, 2018; Kayumov \& Ponnusamy, 2018; Silverman, 1998) and references therein. In the well-established theory of analytic univalent functions, there are several studies on hypergeometric functions associated with classes of analytic functions (See for example Carlson \& Shaffer, 1984; Miller \& Mocanu, 1990; Kwon \& Cho, 2008; Owa \& Srivastava, 1987; Ponnusamy \& Ronning, 1998; Ruscheweyh \& Singh, 1986; Silverman, 1993; Swaminathan, 2004a, 2004b) investigating univalence, starlikeness and other properties of these functions. On the other hand, only some corresponding studies on connections of hypergeometric functions with harmonic mappings have been done (Ahuja, 2008, 2007; Murugusundaramoorthy \& Raina, 2009). Pursuing this line of study, results that bring out connections of hypergeometric functions with a class of harmonic univalent functions considered in (Yalçin \& Öztürk, 2004) are established.

Let $H$ be the class of continuous, complex-valued harmonic functions $f(z)=u+i v$ which map the unit disk $U=\{z \in C:|z|<1\}$ onto a domain $D \subset C$. In fact $u$ and $v$ are real harmonic in $U$. It is well-known (Clunie and Sheil-Small, 1984) that such a harmonic function $f$ can be written as $f=h+\bar{g}$, when $h$ and $g$ are analytic in $U$. It is also known (Clunie and SheilSmall, 1984) that a sufficient condition for $f=h+\bar{g}$ to be locally univalent and sense preserving in $U$ is that $\left|h^{\prime}(z)\right|>\left|g^{\prime}(z)\right|$ in $U$.

Denote by $S_{H}$ the class of functions $f=h+\bar{g}$ which are harmonic univalent and sense preserving in the unit disk $U$ and $f$ normalized by $f(0)=h(0)=f_{z}(0)-1=0 . \quad$ Thus, for $f=h+\bar{g} \in S_{H}$ we may express the analytic functions

\footnotetext{
*Corresponding author: chandra@uthm.edu.my
} 
$h$ and $g$ as

$$
h(z)=z+\sum_{n=2}^{\infty} A_{n} z^{n} \text { and } g(z)=\sum_{n=1}^{\infty} B_{n} z^{n}(1)
$$

where $\left|B_{1}\right|<1$. Note that $S_{H}$ reduces to the class of normalized analytic univalent functions if the co-analytic part $g$ of $f$ is identically zero. If $\phi_{1}$ and $\phi_{2}$ are analytic and $f=h+\bar{g}$ is in $S_{H}$, the convolution or the Hadamard product is defined by

$$
f *\left(\phi_{1}+\overline{\phi_{2}}\right)=h * \phi_{1}+\overline{g * \phi_{2}} .
$$

Let $a, b$ and $c$ be any complex numbers with $c \neq 0,-1,-2,-3, \ldots$. Then the Gauss hypergeometric function written as ${ }_{2} F_{1}(a, b ; c ; z)$ or simply as $F_{c}^{a, b}(z)$ is defined by

$$
F_{c}^{a, b}(z)=\sum_{n=0}^{\infty} \frac{(a)_{n}(b)_{n}}{(c)_{n}(1)_{n}} z^{n}
$$

where $(\lambda)_{n}$ is the Pochhammer symbol given by

$$
(\lambda)_{n}=\left\{\begin{array}{cc}
1, & (n=0) ; \\
\lambda(\lambda+1)(\lambda+2) \ldots(\lambda+n-1), & (n=N) .
\end{array}\right.
$$

Since the hypergeometric series defined in (2) and (3) converges absolutely in $U$, it follows that $F_{c}^{a, b}(z)$ defines a function which is analytic in $U$, provided that $c$ is neither zero nor a negative integer. In fact, $F_{c}^{a, b}(1)$ converges for $\operatorname{Re}(c-a-b>0)$ and is related to the gamma function by

$$
F_{c}^{a, b}(1)=\frac{\Gamma(c) \Gamma(c-a-b)}{\Gamma(c-a) \Gamma(c-b)}, c \neq 0,1,2, \ldots
$$

In particular, the incomplete beta function, related to the Gauss hypergeometric $\varphi(a, c ; z)$, is defined by

$$
\varphi(a, c ; z)=F_{c}^{a, 1}(z)=\sum_{n=0}^{\infty} \frac{(a)_{n}}{(c)_{n}} z^{n+1}
$$

where $z \in U$ and $c \neq 0,1,2, \ldots$
Throughout this paper, let $G(z)=\phi_{1}(z)+\overline{\phi_{2}(z)}$ be a function where $\phi_{1}(z)$ and $\phi_{2}(z)$ are the hypergeometric functions defined by

$\phi_{1}(z):=z F_{c_{1}}^{a_{1}, b_{1}}(z)=z+\sum_{n=2}^{\infty} \frac{\left(a_{1}\right)_{n-1}\left(b_{1}\right)_{n-1}}{\left(c_{1}\right)_{n-1}(1)_{n-1}} z^{n}$

and

$$
\phi_{2}(z):=F_{c_{2}}^{a_{2}, b_{2}}(z)-1=\sum_{n=1}^{\infty} \frac{\left(a_{2}\right)_{n}\left(b_{2}\right)_{n}}{\left(c_{2}\right)_{n}(1)_{n}} z^{n}
$$

where $\left|a_{2} b_{2}\right|<\left|c_{2}\right|$. The following lemma is needed to prove our results.

Lemma 1. (Ahuja, 2008) If $a, b, c>0$, then

$$
\sum_{n=1}^{\infty} n \frac{(a)_{n}(b)_{n}}{(c)_{n}(1)_{n}}=\frac{a b}{c-a-b-1} F_{c}^{a, b}(1)
$$

if $c>a+b+1$

$$
\begin{aligned}
\sum_{n=1}^{\infty} n^{2} & \frac{(a)_{n}(b)_{n}}{(c)_{n}(1)_{n}} \\
\quad & {\left[\frac{(a)_{2}(b)_{2}}{(c-a-b-2)_{2}}+\frac{a b}{c-a-b-1}\right] F_{c}^{a, b} }
\end{aligned}
$$

$$
\text { if } c>a+b+2 \text {. }
$$

Based on the study in (Yalçin \& Öztürk, 2004), for $\alpha \geq 0$ and $0 \leq \beta<1$, we define a class $H P(\alpha, \beta)$ of harmonic functions of the form (1) satisfying the condition

$$
\operatorname{Re}\left\{\alpha z\left[h^{\prime \prime}(z)+g^{\prime \prime}(z)\right]+\left[h^{\prime}(z)+g^{\prime}(z)\right]\right\}>\beta .
$$

Lemma 2. If $f=h+\bar{g}$ is given by (1) and

$\sum_{n=1}^{\infty} n[\alpha(n-1)+1]\left(\left|A_{n}\right|+\left|B_{n}\right|\right) \leq 2-\beta$

where $0 \leq\left|B_{1}\right|<1-\beta, A_{1}=1, \alpha \geq 0$ and $0 \leq \beta<1$, then $f$ is harmonic univalent and sense preserving in $U$ and $f \in H P(\alpha, \beta)$.

Proof. The proof of this lemma is on lines similar to the proof of Theorem 2.1 in (Yalçin \& Öztürk, 2004). 


\section{MAIN RESULTS}

Theorem 1. If $a_{j}, b_{j}>0$ and $c_{j}>a_{j}+b_{j}+2$ for $j=1,2$, then a sufficient condition for $G=\phi_{1}+\overline{\phi_{2}}$ to be harmonic univalent in $U$ and $G \in H P(\alpha, \beta)$, is that

$$
\begin{aligned}
& {\left[\frac{\alpha\left(a_{1}\right)_{2}\left(b_{1}\right)_{2}}{\left(c_{1}-a_{1}-b_{1}-2\right)_{2}}+\frac{a_{1} b_{1}(2 \alpha+1)}{c_{1}-a_{1}-b_{1}-1}+1\right] F_{c_{1}}^{a_{1}, b_{1}}(1)} \\
& +\left[\frac{\alpha\left(a_{2}\right)_{2}\left(b_{2}\right)_{2}}{\left(c_{2}-a_{2}-b_{2}-2\right)_{2}}+\frac{a_{2} b_{2}}{c_{2}-a_{2}-b_{2}-1}\right] F_{c_{2}}^{a_{2}, b_{2}}(1)
\end{aligned}
$$

$$
\leq 2-\beta
$$

where $\alpha \geq 0$ and $0 \leq \beta<1$.

Proof. When the condition (11) holds for the coefficients of $G=\phi_{1}+\bar{\phi}_{2}$, by using (10) it is enough to prove that

$$
\sum_{n=1}^{\infty} n(\alpha(n-1)+1)\left[\frac{\left(a_{1}\right)_{n-1}\left(b_{1}\right)_{n-1}}{\left(c_{1}\right)_{n-1}(1)_{n-1}}+\frac{\left(a_{2}\right)_{n}\left(b_{2}\right)_{n}}{\left(c_{2}\right)_{n}(1)_{n}}\right]
$$

$\leq 2-\beta$.

(12)

Write the left side of equality (12) as

$$
\begin{aligned}
& \alpha \sum_{n=1}^{\infty} n(n-1) \frac{\left(a_{1}\right)_{n-1}\left(b_{1}\right)_{n-1}}{\left(c_{1}\right)_{n-1}(1)_{n-1}} \\
& +\alpha \sum_{n=1}^{\infty} n(n-1) \frac{\left(a_{2}\right)_{n}\left(b_{2}\right)_{n}}{\left(c_{2}\right)_{n}(1)_{n}} \\
& +\sum_{n=1}^{\infty} n \frac{\left(a_{1}\right)_{n-1}\left(b_{1}\right)_{n-1}}{\left(c_{1}\right)_{n-1}(1)_{n-1}}+\sum_{n=1}^{\infty} n \frac{\left(a_{2}\right)_{n}\left(b_{2}\right)_{n}}{\left(c_{2}\right)_{n}(1)_{n}} \\
= & \alpha \sum_{n=1}^{\infty}\left[(n-1)^{2}+(n-1)\right] \frac{\left(a_{1}\right)_{n-1}\left(b_{1}\right)_{n-1}}{\left(c_{1}\right)_{n-1}(1)_{n-1}} \\
& +\alpha \sum_{n=1}^{\infty}\left(n^{2}-n\right) \frac{\left(a_{2}\right)_{n}\left(b_{2}\right)_{n}}{\left(c_{2}\right)_{n}(1)_{n}} \\
& +\sum_{n=1}^{\infty}(n-1+1) \frac{\left(a_{1}\right)_{n-1}\left(b_{1}\right)_{n-1}}{\left(c_{1}\right)_{n-1}(1)_{n-1}}+\sum_{n=1}^{\infty} n \frac{\left(a_{2}\right)_{n}\left(b_{2}\right)_{n}}{\left(c_{2}\right)_{n}(1)_{n}} \\
= & \alpha \sum_{n=1}^{\infty} n^{2} \frac{\left(a_{1}\right)_{n}\left(b_{1}\right)_{n}}{\left(c_{1}\right)_{n}(1)_{n}}+(\alpha+1) \sum_{n=1}^{\infty} n \frac{\left(a_{1}\right)_{n}\left(b_{1}\right)_{n}}{\left(c_{1}\right)_{n}(1)_{n}} \\
& +\sum_{n=0}^{\infty} \frac{\left(a_{1}\right)_{n}\left(b_{1}\right)_{n}}{\left(c_{1}\right)_{n}(1)_{n}}+\alpha \sum_{n=1}^{\infty} n^{2} \frac{\left(a_{2}\right)_{n}\left(b_{2}\right)_{n}}{\left(c_{2}\right)_{n}(1)_{n}} \\
& -(\alpha-1) \sum_{n=1}^{\infty} n \frac{\left(a_{2}\right)_{n}\left(b_{2}\right)_{n}}{\left(c_{2}\right)_{n}(1)_{n}}
\end{aligned}
$$

$$
\begin{aligned}
= & \alpha\left[\frac{\left(a_{1}\right)_{2}\left(b_{1}\right)_{2}}{\left(c_{1}-a_{1}-b_{1}-2\right)_{2}}+\frac{a_{1} b_{1}}{c_{1}-a_{1}-b_{1}-1}\right] F_{c_{1}}^{a_{1}, b_{1}}(1) \\
& +(\alpha+1) \frac{a_{1} b_{1}}{c_{1}-a_{1}-b_{1}-1} F_{c_{1}}^{a_{1}, b_{1}}(1)+F_{c_{1}}^{a_{1}, b_{1}}(1) \\
& +\alpha\left[\frac{\left(a_{2}\right)_{2}\left(b_{2}\right)_{2}}{\left(c_{2}-a_{2}-b_{2}-2\right)_{2}}+\frac{a_{2} b_{2}}{c_{2}-a_{2}-b_{2}-1}\right] F_{c_{2}}^{a_{2}, b_{2}}(1) \\
& -(\alpha-1) \frac{a_{2} b_{2}}{c_{2}-a_{2}-b_{2}-1} F_{c_{2}}^{a_{2}, b_{2}}(1),
\end{aligned}
$$

by an application of equation (8) and (9). This yield (11). In order to prove that $G$ is locally univalent and sensepreserving in $U$, it is sufficient to show that $\left|\phi_{1}^{\prime}(z)\right|>\left|\phi_{2}^{\prime}(z)\right|$, 


$$
\begin{aligned}
& \left|\phi_{1}^{\prime}(z)\right|=\left|1+\sum_{n=2}^{\infty} n \frac{\left(a_{1}\right)_{n-1}\left(b_{1}\right)_{n-1}}{\left(c_{1}\right)_{n-1}(1)_{n-1}} z^{n-1}\right| \\
& >1-\sum_{n=2}^{\infty}(n-1) \frac{\left(a_{1}\right)_{n-1}\left(b_{1}\right)_{n-1}}{\left(c_{1}\right)_{n-1}(1)_{n-1}} \\
& -\sum_{n=2}^{\infty} \frac{\left(a_{1}\right)_{n-1}\left(b_{1}\right)_{n-1}}{\left(c_{1}\right)_{n-1}(1)_{n-1}} \\
& =1-\frac{a_{1} b_{1}}{c_{1}} \sum_{n=1}^{\infty} \frac{\left(a_{1}+1\right)_{n-1}\left(b_{1}+1\right)_{n-1}}{\left(c_{1}+1\right)_{n-1}(1)_{n-1}} \\
& -\sum_{n=1}^{\infty} \frac{\left(a_{1}\right)_{n}\left(b_{1}\right)_{n}}{\left(c_{1}\right)_{n}(1)_{n}} \\
& \geq 2-\beta-\left[\begin{array}{c}
\frac{\alpha\left(a_{1}\right)_{2}\left(b_{1}\right)_{2}}{\left(c_{1}-a_{1}-b_{1}-2\right)_{2}} \\
+\frac{a_{1} b_{1}(2 \alpha+1)}{c_{1}-a_{1}-b_{1}-1}+1
\end{array}\right] \\
& \times F_{c_{1}}^{a_{1}, b_{1}}(1) \\
& \geq\left[\begin{array}{c}
\frac{\alpha\left(a_{2}\right)_{2}\left(b_{2}\right)_{2}}{\left(c_{2}-a_{2}-b_{2}-2\right)_{2}} \\
+\frac{a_{2} b_{2}}{c_{2}-a_{2}-b_{2}-1}
\end{array}\right] F_{c_{2}}^{a_{2}, b_{2}}(1) \\
& \geq \frac{a_{2} b_{2}}{c_{2}} \frac{\Gamma\left(c_{2}+1\right) \Gamma\left(c_{2}-a_{2}-b_{2}-1\right)}{\Gamma\left(c_{2}-a_{2}\right) \Gamma\left(c_{2}-b_{2}\right)} \\
& =\sum_{n=0}^{\infty} \frac{\left(a_{2}\right)_{n+1}\left(b_{2}\right)_{n+1}}{\left(c_{2}\right)_{n+1}(1)_{n}} \\
& \geq\left|\sum_{n=1}^{\infty} n \frac{\left(a_{2}\right)_{n}\left(b_{2}\right)_{n}}{\left(c_{2}\right)_{n}(1)_{n}} z^{n-1}\right|=\left|\phi_{2}^{\prime}(z)\right| \text {. }
\end{aligned}
$$

In fact, for $\left|z_{1}\right| \leq\left|z_{2}\right|<1$, we have

$$
\begin{aligned}
& \left|G\left(z_{1}\right)-G\left(z_{2}\right)\right| \\
\geq & \left|\phi_{1}\left(z_{1}\right)-\phi_{1}\left(z_{2}\right)\right|-\left|\phi_{2}\left(z_{1}\right)-\phi_{2}\left(z_{2}\right)\right| \\
= & \left|\left(z_{1}-z_{2}\right)+\sum_{n=2}^{\infty} \frac{\left(a_{1}\right)_{n-1}\left(b_{1}\right)_{n-1}}{\left(c_{1}\right)_{n-1}(1)_{n-1}}\left(z_{1}^{n}-z_{2}^{n}\right)\right| \\
& -\left|\sum_{n=1}^{\infty} \frac{\left(a_{2}\right)_{n}\left(b_{2}\right)_{n}}{\left(c_{2}\right)_{n}(1)_{n}}\left(z_{1}^{n}-z_{2}^{n}\right)\right| \\
\geq \mid & \mid \begin{array}{c}
\left.1-\frac{a_{2} b_{2}}{c_{2}}-z_{2}|| \begin{array}{c}
\left(a_{1}\right)_{n-1}\left(b_{1}\right)_{n-1} \\
\left(c_{1}\right)_{n-1}(1)_{n-1} \\
-\sum_{n=2}^{\infty} n \\
+\frac{\left(a_{2}\right)_{n}\left(b_{2}\right)_{n}}{\left(c_{2}\right)_{n}(1)_{n}}
\end{array}\right)\left|z_{2}\right|^{n-1} \\
2-\beta \\
=
\end{array} \\
& \times\left[\begin{array}{c}
z_{1}-z_{2} \mid \\
-\sum_{n=1}^{\infty} n(\alpha(n-1)+1)\left[\begin{array}{c}
\left(a_{1}\right)_{n-1}\left(b_{1}\right)_{n-1} \\
\left(c_{1}\right)_{n-1}(1)_{n-1} \\
+\frac{\left(a_{2}\right)_{n}\left(b_{2}\right)_{n}}{\left(c_{2}\right)_{n}(1)_{n}}
\end{array}\right]
\end{array}\right]
\end{aligned}
$$

In view of (12), $\left|G\left(z_{1}\right)-G\left(z_{2}\right)\right| \geq 0$ which shows that $G$ is univalent in $U$. This completes the proof.

Denote by $H T(\alpha, \beta)=H P(\alpha, \beta) \bigcap T_{H}$ where $T_{H}$ [16], is the class of harmonic functions $f=h+\bar{g}$ of the form

$$
h(z)=z-\sum_{n=2}^{\infty} A_{n} z^{n} \text { and } g(z)=-\sum_{n=1}^{\infty} B_{n} z^{n}
$$

where $A_{n}, B_{n} \geq 0$ for $n=1,2, \ldots$ and $B_{1}<1$.

Lemma 3. If $f=h+\bar{g}$ is given by (13), then $f \in H T(\alpha, \beta)$ if and only if

$$
\sum_{n=1}^{\infty} n[\alpha(n-1)+1]\left(A_{n}+B_{n}\right) \leq 2-\beta
$$

where $\alpha \geq 0,0 \leq \beta<1, A_{1}=1$ and $0 \leq B_{1}<1-\beta$.

The sufficiency of this result is from Lemma 2 and the proof of necessity is on lines similar to the proof of Theorem 2.2 in (Yalçin \& Öztürk, 2004). Define 


$$
\begin{aligned}
G_{1}(z)= & z\left(2-\frac{\phi_{1}(z)}{z}\right)-\overline{\phi_{2}(z)} \\
= & z-\sum_{n=2}^{\infty} \frac{\left(a_{1}\right)_{n-1}\left(b_{1}\right)_{n-1}}{\left(c_{1}\right)_{n-1}(1)_{n-1}} z^{n} \\
& -\frac{\sum_{n=1}^{\infty} \frac{\left(a_{2}\right)_{n}\left(b_{2}\right)_{n}}{\left(c_{2}\right)_{n}(1)_{n}} z^{n}}{}
\end{aligned}
$$

on using (6) and (7). Clearly $G_{1} \in T_{H}$. Theorem 2. Let $\alpha \geq 0,0 \leq \beta<1, a_{j}, b_{j}>0, c_{j}>a_{j}+b_{j}+2, \quad$ for $j=1,2$ and $a_{2} b_{2}<c_{2} . G_{1}$ is in $H T(\alpha, \beta)$ if and only if (11) holds.

Proof. If $G_{1} \in H T(\alpha, \beta)$, then $G_{1}$ satisfies (12) by Lemma 3 and hence (11) holds.

Theorem 3. Let $0 \leq \beta<1, a_{j}, b_{j}>0, c_{j}>a_{j}$ $+b_{j}+1$, for $j=1,2$ and $a_{2} b_{2}<c_{2}$. A necessary and sufficient condition such that $f *\left(\phi_{1}+\overline{\phi_{2}}\right) \in H T(\alpha, \beta)$ for $f \in H T(\alpha, \beta)$ is that

$$
F_{c_{1}}^{a_{1}, b_{1}}(1)+F_{c_{2}}^{a_{2}, b_{2}}(1) \leq 3
$$

where $\phi_{1}, \phi_{2}$ are as defined, respectively, by (6) and (7).

Proof. Let $f=h+\bar{g} \in H T(\alpha, \beta)$, where $h$ and $g$ are given by (13). Then

$$
\begin{aligned}
\left(f *\left(\phi_{1}+\overline{\phi_{2}}\right)\right)(z)= & h(z) * \phi_{1}(z)+\overline{g(z) * \phi_{2}(z)} \\
= & z-\sum_{n=2}^{\infty} \frac{\left(a_{1}\right)_{n-1}\left(b_{1}\right)_{n-1}}{\left(c_{1}\right)_{n-1}(1)_{n-1}} A_{n} z^{n} \\
& -\overline{\sum_{n=1}^{\infty} \frac{\left(a_{2}\right)_{n}\left(b_{2}\right)_{n}}{\left(c_{2}\right)_{n}(1)_{n}} B_{n} z^{n}} .
\end{aligned}
$$

In view of Lemma 3, we need to prove that $\left(f *\left(\phi_{1}+\overline{\phi_{2}}\right)\right) \in H T(\alpha, \beta)$ if and only if

$$
\sum_{n=1}^{\infty} n(\alpha(n-1)+1)\left[\begin{array}{c}
\frac{\left(a_{1}\right)_{n-1}\left(b_{1}\right)_{n-1}}{\left(c_{1}\right)_{n-1}(1)_{n-1}} A_{n} \\
+\frac{\left(a_{2}\right)_{n}\left(b_{2}\right)_{n}}{\left(c_{2}\right)_{n}(1)_{n}} B_{n}
\end{array}\right]
$$

$\leq 2-\beta$

As an application of Lemma 3, we have

$$
A_{n} \leq \frac{1-\beta}{n(\alpha(n-1)+1)}, n=2,3, \ldots
$$

and

$$
B_{n} \leq \frac{1-\beta}{n(\alpha(n-1)+1)}, n=1,2, \ldots .
$$

Therefore, the left side of (15) is bounded above by

$$
\begin{aligned}
& \sum_{n=2}^{\infty}(1-\beta) \frac{\left(a_{1}\right)_{n-1}\left(b_{1}\right)_{n-1}}{\left(c_{1}\right)_{n-1}(1)_{n-1}}+\sum_{n=1}^{\infty}(1-\beta) \frac{\left(a_{2}\right)_{n}\left(b_{2}\right)_{n}}{\left(c_{2}\right)_{n}(1)_{n}} \\
= & (1-\beta)\left[\sum_{n=1}^{\infty} \frac{\left(a_{1}\right)_{n}\left(b_{1}\right)_{n}}{\left(c_{1}\right)_{n}(1)_{n}}+\sum_{n=1}^{\infty} \frac{\left(a_{2}\right)_{n}\left(b_{2}\right)_{n}}{\left(c_{2}\right)_{n}(1)_{n}}\right] \\
= & (1-\beta)\left[F_{c_{1}}^{a_{1}, b_{1}}(1)+F_{c_{2}}^{a_{2}, b_{2}}(1)-2\right] .
\end{aligned}
$$

The last expression is bounded above by $(1-\beta)$ if and only if (14) is satisfied. This proves (15) and the results follows.

Theorem 4. If $a_{j}, b_{j}>0$ and $c_{j}>a_{j}+b_{j}+1$ for $j=1,2$, then a sufficient condition for a function

$$
G_{2}(z)=\int_{0}^{z} F_{c_{1}}^{a_{1}, b_{1}}(t) d t+\overline{\int_{0}^{z}\left[F_{c_{2}}^{a_{2}, b_{2}}(t)-1\right] d t}
$$

to be in $H P(\alpha, \beta)$ is that

$$
\begin{aligned}
& \left(\frac{\alpha\left(a_{1} b_{1}\right)}{c_{1}-a_{1}-b_{1}-1}+1\right) F_{c_{1}}^{a_{1}, b_{1}}(1) \\
& \quad+\left(\frac{\alpha\left(a_{2} b_{2}\right)}{c_{2}-a_{2}-b_{2}-1}+1\right) F_{c_{2}}^{a_{2}, b_{2}}(1) \\
& \leq 3-\beta
\end{aligned}
$$

where $\alpha \geq 0$ and $0 \leq \beta<1$.

Proof. In view of Lemma 2, the function

$$
\begin{aligned}
G_{2}(z)= & z+\sum_{n=2}^{\infty} \frac{\left(a_{1}\right)_{n-1}\left(b_{1}\right)_{n-1}}{\left(c_{1}\right)_{n-1}(1)_{n}} z^{n} \\
& +\sum_{n=2}^{\infty} \frac{\left(a_{2}\right)_{n-1}\left(b_{2}\right)_{n-1}}{\left(c_{2}\right)_{n-1}(1)_{n}} z^{n}
\end{aligned}
$$

is in $H P(\alpha, \beta)$ if

$$
\sum_{n=2}^{\infty} n(\alpha(n-1)+1)\left[\begin{array}{c}
\frac{\left(a_{1}\right)_{n-1}\left(b_{1}\right)_{n-1}}{\left(c_{1}\right)_{n-1}(1)_{n}} \\
+\frac{\left(a_{2}\right)_{n-1}\left(b_{2}\right)_{n-1}}{\left(c_{2}\right)_{n-1}(1)_{n}}
\end{array}\right]
$$

$\leq 1-\beta$.

By a simple computation we obtain 


$$
\begin{aligned}
& \sum_{n=2}^{\infty} n(\alpha(n-1)+1)\left[\begin{array}{c}
\frac{\left(a_{1}\right)_{n-1}\left(b_{1}\right)_{n-1}}{\left(c_{1}\right)_{n-1}(1)_{n}} \\
+\frac{\left(a_{2}\right)_{n-1}\left(b_{2}\right)_{n-1}}{\left(c_{2}\right)_{n-1}(1)_{n}}
\end{array}\right] \\
& =\sum_{n=1}^{\infty}(\alpha n+1)\left[\frac{\left(a_{1}\right)_{n}\left(b_{1}\right)_{n}}{\left(c_{1}\right)_{n}(1)_{n}}+\frac{\left(a_{2}\right)_{n}\left(b_{2}\right)_{n}}{\left(c_{2}\right)_{n}(1)_{n}}\right] .
\end{aligned}
$$

The result follows from an application of Lemma 1.

Theorem 5. If $a_{1}, b_{1}>-1, c_{1}>0, a_{1} b_{1}<0$, $a_{2}>0, b_{2}>0$, and $c_{j}>a_{j}+b_{j}+2, j=1,2$, then

$$
G_{3}(z)=\int_{0}^{z} F_{c_{1}}^{a_{1}, b_{1}}(t) d t-\overline{\int_{0}^{z}\left[F_{c_{2}}^{a_{2}, b_{2}}(t)-1\right] d t}
$$

to be in $H T(\alpha, \beta)$ if and only if

$$
\begin{aligned}
& \left(\frac{\alpha\left(a_{1} b_{1}\right)}{c_{1}-a_{1}-b_{1}-1}+1\right) F_{c_{1}}^{a_{1}, b_{1}}(1) \\
& -\left(\frac{\alpha\left(a_{2} b_{2}\right)}{c_{2}-a_{2}-b_{2}-1}+1\right) F_{c_{2}}^{a_{2}, b_{2}}(1)+1 \geq \beta
\end{aligned}
$$

where $\alpha \geq 0$ and $0 \leq \beta<1$.

Proof. We write

$$
\begin{aligned}
G_{3}(z)= & z-\frac{\left|a_{1} b_{1}\right|}{c_{1}} \sum_{n=2}^{\infty} \frac{\left(a_{1}+1\right)_{n-2}\left(b_{1}+1\right)_{n-2}}{\left(c_{1}+1\right)_{n-2}(1)_{n}} z^{n} \\
& -\sum_{n=2}^{\infty} \frac{\left(a_{2}\right)_{n-1}\left(b_{2}\right)_{n-1}}{\left(c_{2}\right)_{n-1}(1)_{n}} z^{n} .
\end{aligned}
$$

In view of Lemma 3 it is sufficient to show that

$$
\sum_{n=2}^{\infty} n(\alpha(n-1)+1)\left[\begin{array}{c}
\frac{\left|a_{1} b_{1}\right|}{c_{1}} \frac{\left(a_{1}+1\right)_{n-2}\left(b_{1}+1\right)_{n-2}}{\left(c_{1}+1\right)_{n-2}(1)_{n}} \\
+\frac{\left(a_{2}\right)_{n-1}\left(b_{2}\right)_{n-1}}{\left(c_{2}\right)_{n-1}(1)_{n}}
\end{array}\right]
$$

$\leq 1-\beta$.

By a routine computation (17) can be written as

$$
\begin{aligned}
& \alpha \sum_{n=1}^{\infty} \frac{\left|a_{1} b_{1}\right|}{c_{1}} \frac{\left(a_{1}+1\right)_{n-1}\left(b_{1}+1\right)_{n-1}}{\left(c_{1}+1\right)_{n-1}(1)_{n-1}} \\
& +\alpha \sum_{n=1}^{\infty} n \frac{\left(a_{2}\right)_{n}\left(b_{2}\right)_{n}}{\left(c_{2}\right)_{n}(1)_{n}} \\
& +\sum_{n=1}^{\infty} \frac{\left|a_{1} b_{1}\right|}{c_{1}} \frac{\left(a_{1}+1\right)_{n-1}\left(b_{1}+1\right)_{n-1}}{\left(c_{1}+1\right)_{n-1}(1)_{n}}+\sum_{n=1}^{\infty} \frac{\left(a_{2}\right)_{n}\left(b_{2}\right)_{n}}{\left(c_{2}\right)_{n}(1)_{n}} \\
& \leq(1-\beta) .
\end{aligned}
$$

Or equivalently

$$
\begin{aligned}
& \alpha \sum_{n=0}^{\infty} \frac{\left(a_{1}+1\right)_{n}\left(b_{1}+1\right)_{n}}{\left(c_{1}+1\right)_{n}(1)_{n}}+\frac{\alpha c_{1}}{\left|a_{1} b_{1}\right|} \sum_{n=1}^{\infty} n \frac{\left(a_{2}\right)_{n}\left(b_{2}\right)_{n}}{\left(c_{2}\right)_{n}(1)_{n}} \\
& +\sum_{n=0}^{\infty} \frac{\left(a_{1}+1\right)_{n}\left(b_{1}+1\right)_{n}}{\left(c_{1}+1\right)_{n}(1)_{n+1}}+\frac{c_{1}}{\left|a_{1} b_{1}\right|} \sum_{n=1}^{\infty} \frac{\left(a_{2}\right)_{n}\left(b_{2}\right)_{n}}{\left(c_{2}\right)_{n}(1)_{n}} \\
& \leq \frac{c_{1}(1-\beta)}{\left|a_{1} b_{1}\right|} .
\end{aligned}
$$

But, this is equivalent to

$$
\begin{aligned}
& \frac{\alpha c_{1}}{a_{1} b_{1}} \sum_{n=1}^{\infty} n \frac{\left(a_{1}\right)_{n}\left(b_{1}\right)_{n}}{\left(c_{1}\right)_{n}(1)_{n}}+\frac{\alpha c_{1}}{\left|a_{1} b_{1}\right|} \sum_{n=1}^{\infty} n \frac{\left(a_{2}\right)_{n}\left(b_{2}\right)_{n}}{\left(c_{2}\right)_{n}(1)_{n}} \\
& +\frac{c_{1}}{a_{1} b_{1}} \sum_{n=1}^{\infty} \frac{\left(a_{1}\right)_{n}\left(b_{1}\right)_{n}}{\left(c_{1}\right)_{n}(1)_{n}}+\frac{c_{1}}{\left|a_{1} b_{1}\right|} \sum_{n=1}^{\infty} \frac{\left(a_{2}\right)_{n}\left(b_{2}\right)_{n}}{\left(c_{2}\right)_{n}(1)_{n}} \\
& \leq \frac{c_{1}(1-\beta)}{\left|a_{1} b_{1}\right|},
\end{aligned}
$$

which yields

$$
\begin{aligned}
& \left(\frac{\alpha\left(a_{1} b_{1}\right)}{c_{1}-a_{1}-b_{1}-1}+1\right) F_{c_{1}}^{a_{1}, b_{1}}(1) \\
& \quad-\left(\frac{\alpha\left(a_{2} b_{2}\right)}{c_{2}-a_{2}-b_{2}-1}+1\right) F_{c_{2}}^{a_{2}, b_{2}}(1) \geq-1+\beta .
\end{aligned}
$$

This completes the proof.

In particular, the results parallel to Theorems 3, 6, 7 and 8 may also be obtained for the incomplete beta function $\varphi(a, c ; z)$ as defined by (4) and (5). Let

$$
\phi_{1}(z)=\varphi\left(a_{1}, c_{1} ; z\right)=z+\sum_{n=2}^{\infty} \frac{\left(a_{1}\right)_{n-1}}{\left(c_{1}\right)_{n-1}} z^{n}
$$

and

$$
\phi_{2}(z)=\frac{1}{z} \varphi\left(a_{2}, c_{2} ; z\right)-1=\sum_{n=1}^{\infty} \frac{\left(a_{2}\right)_{n}}{\left(c_{2}\right)_{n}} z^{n}
$$

where $\left|a_{2}\right|<\left|c_{2}\right|$. Making use of

$$
F_{c_{1}}^{a_{1}, 1}(1)=\frac{c_{1}-1}{c_{1}-a_{1}-1} \text { and } F_{c_{2}}^{a_{2}, 1}(1)-1=\frac{a_{2}}{c_{2}-a_{2}-1}
$$

the following theorems are obtained.

Theorem 6. If $a_{j}>0$ and $c_{j}>a_{j}+3$ for $j=1,2$, then a sufficient condition for $G=\phi_{1}+\overline{\phi_{2}}$ to be harmonic univalent in $U$ with $\phi_{1}+\overline{\phi_{2}} \in H P(\alpha, \beta)$, is that 
$\left[\frac{2 \alpha\left(a_{1}\right)_{2}}{\left(c_{1}-a_{1}-3\right)_{2}}+\frac{2 \alpha a_{1}+c_{1}-2}{c_{1}-a_{1}-2}\right] \frac{c_{1}-1}{c_{1}-a_{1}-1}$

$+\left[\frac{2 \alpha\left(a_{2}\right)_{2}}{\left(c_{2}-a_{2}-3\right)_{2}}+\frac{a_{2}}{c_{2}-a_{2}-2}\right] \frac{c_{2}-1}{c_{2}-a_{2}-1}$

$\leq 2-\beta$

where $\alpha \geq 0$ and $0 \leq \beta<1$.

Note that the condition (18) is necessary and sufficient for $G=\phi_{1}+\overline{\phi_{2}}$ to be in $H T(\alpha, \beta)$.

Theorem 7. Let $0 \leq \beta<1, a_{j}>0, c_{j}>a_{j}+2$, for $j=1,2$ and $a_{2}<c_{2}$. A necessary and sufficient condition such that $f *\left(\phi_{1}+\overline{\phi_{2}}\right) \in H T(\alpha, \beta)$ for $f \in H T(\alpha, \beta)$ is that

$$
\frac{c_{1}-1}{c_{1}-a_{1}-1}+\frac{c_{2}-1}{c_{2}-a_{2}-1} \leq 3-\beta .
$$

Theorem 8. If $a_{j}>0$ and $c_{j}>a_{j}+2$ for $j=1,2$, then sufficient condition for

$$
\int_{0}^{z} \varphi\left(a_{1}, c_{1} ; t\right) d t+\overline{\int_{0}^{z}\left[\varphi\left(a_{2}, c_{2} ; t\right)-1\right] d t}
$$

is in $H P(\alpha, \beta)$ is

$\left(\frac{\alpha a_{1}}{c_{1}-a_{1}-2}+1\right) \frac{c_{1}-1}{c_{1}-a_{1}-1}+\left(\frac{\alpha a_{2}}{c_{2}-a_{2}-2}+1\right) \frac{c_{2}-1}{c_{2}-a_{2}-1}$

$\leq 3-\beta$

where $\alpha \geq 0$ and $0 \leq \beta<1$.

Theorem 9. If $a_{1}>-1, c_{1}>0, a_{1}<0, a_{2}>0$, and $c_{j}>a_{j}+3, j=1,2$, then

$$
\int_{0}^{z} \varphi\left(a_{1}, c_{1} ; t\right) d t-\overline{\int_{0}^{z}\left[\varphi\left(a_{2}, c_{2} ; t\right)-1\right] d t}
$$

is in $H T(\alpha, \beta)$ if and only if

$\left(\frac{\alpha a_{1}}{c_{1}-a_{1}-2}+1\right) \frac{c_{1}-1}{c_{1}-a_{1}-1}-\left(\frac{\alpha a_{2}}{c_{2}-a_{2}-2}+1\right) \frac{c_{2}-1}{c_{2}-a_{2}-1}+1$

$\geq \beta$

where $\alpha \geq 0$ and $0 \leq \beta<1$.

Note that Theorems 3.1 and 3.5 in (Al-Khal \& AlKharsani, 2006) are respectively obtained from Theorem 8 and Theorem 4.

\section{ACKNOWLEDGEMENT}

The first author gratefully acknowledges support from FRGS research grant (1634) from the Ministry of Education (MOE), Malaysia and UTHM Contract Grant (U428). 


\section{REFERENCES}

Ahuja O. P. (2005). Planar harmonic univalent and related mappings, J. Inequal. Pure Appl. Math., 6(4), 1-18.

Ahuja O. P. (2007). Planar harmonic convolution operators generated by hypergeometric functions, Integral Transforms Spec. Funct., 18(3), 165-177.

Ahuja O. P. (2008). Connections between various subclasses of planar harmonic mappings involving hypergeometric functions, Appl. Math. Comput., 198(1), 305-316.

Al-Khal R. and Al-Kharsani H. A. (2006). Harmonic hypergeometric functions, Tamkang J. Math., 37(3), $273-283$.

Carlson B. C. and Shaffer D. B. (1984). Starlike and prestarlike hypergeometric functions, SIAM J. Math. Anal., 15(4), 737-745.

Clunie J. and Sheil-Small T. (1984). Harmonic univalent functions, Ann. Acad. Sci. Fenn. Ser. A I Math., 9, 325 .

Duren P. (2004). Harmonic mappings in the plane, 156, Cambridge Univ. Press, Cambridge.

Liu G. and Ponnusamy S. (2018). Uniformly locally univalent harmonic mappings associated with the pre-Schwarzian norm, Indag. Math. (N.S.), 29(2), $752-778$.

Kayumov I. R. and Ponnusamy S. (2018). Bohr's inequalities for the analytic functions with lacunary series and harmonic functions, J. Math. Anal. Appl., 465(2), 857-871.

Miller S. S. and Mocanu P. T. (1990). Univalence of Gaussian and confluent hypergeometric functions, Proc. Amer. Math. Soc., 110(2), 333-342.

Murugusundaramoorthy G. and Raina R. K. (2009). On a subclass of harmonic functions associated with Wright's generalized hypergeometric functions, Hacet. J. Math. Stat., 38(2), 129-136.

Kwon O. S. and. Cho N. E. (2008). Starlike and convex properties for hypergeometric functions, Int. J. Math. Math. Sci., 159029-1

Owa S. and Srivastava H. M. (1987). Univalent and starlike generalized hypergeometric functions, Canad. J. Math., 39(5), 1057-1077.

Ponnusamy S. and Rønning F. (1998). Starlikeness properties for convolutions involving hypergeometric series, Ann. Univ. Mariae Curie-Skłodowska Sect. A, 52(1), 141-155.

Ruscheweyh S. and Singh V. (1986). On the order of starlikeness of hypergeometric functions, J. Math. Anal. Appl., 113(1), 1-11.

Silverman H. (1993). Starlike and convexity properties for hypergeometric functions, J. Math. Anal. Appl., 172(2), 574-581.

Silverman H. (1998). Harmonic univalent functions with negative coefficients, J. Math. Anal. Appl., 220(1), 283-289.

Sheil-Small T. (1990). Constants for planar harmonic mappings, J. London Math. Soc. (2) 2(2), 237-248.

Swaminathan A. (2004a). Certain sufficiency conditions on Gaussian hypergeometric functions. J. Inequal. Pure Appl. Math., 5(4), 1-10.

Swaminathan A. (2004b). Hypergeometric functions in the parabolic domain, Tamsui Oxf. J. Math. Sci., 20(1), 116.

Yalçin S. and Öztürk M. (2004). A new subclass of complex harmonic functions, Math. Inequal. Appl., 7(1), 5561. 